\title{
Experimental Study on Magnetic Induction Properties of PVC Pipe in Electronic Anti-Fouling Technology
}

\author{
Bingcheng Liu, Guanlin Li, Yangyang Li, Hao Sun, Aiping Cheng, Wenguang Jia* \\ Qingdao University of Science Technology, Qingdao City, Shandong Province, China
}

Received: 31 July 2017

Accepted: 25 December 2017

\begin{abstract}
Magnetic induction intensity is a crucial factor for determining the effect of electronic anti-fouling technology, while current is an important factor in determining the strength of magnetic induction intensity. The series resistance method is used in an electronic anti-fouling system in order to get the current value in the coil wrapped around the PVC pipe. The peak current in the circuit is obtained by measuring the voltage crossing series resistance, and the magnetic induction intensity in the midpoint of the solenoid coil is calculated by formula. Then a series of experiments are carried out to explore the change rule of current and magnetic induction intensity with respect to frequency, turns, and diameter. At the end of experiments, the results show that current in the coil decreases with the increase of frequency, number of turns, and pipe diameter. Magnetic induction intensity basically does not change with the increase of turns, and it decreases as the frequency and the diameter increase. Through the analysis of experimental data, the excellent processing parameter of the electronic anti-fouling technology is put forward, which provides guidance for the design and manufacture of the control unit.
\end{abstract}

Keywords: electronic anti-fouling technology, current, magnetic induction, PVC pipe, excellent processing parameter

\section{Introduction}

Water is an important heat transfer medium in heat exchangers [1]. When hard water circulates in the heat transfer equipment, the sparingly soluble salts in the water will produce scale on the surface of the heat transfer equipment, which is traditionally called "fouling" [2]. When scale generates in the surface of heat transfer equipment, at least 2 inevitable disadvantages associated with scales occur. Heat transfer efficiency will be reduced

*e-mail: lyy64825@126.com in the heat transfer equipment due to the small thermal conductivity of scale. Even if a thin coating of scale exists in the heat transfer surface, it will greatly reduce the flow rate of water in the heat exchanger equipment and increase the pressure drop in the pipe [2-8]. In addition, if there is a high heat flux in the heat exchanger, scale can even lead to some local hot spots, which could result in equipment damage or suspended production [3].

Generally, there are 2 ways to deal with scales: chemical and physical [5-8]. When a chemical descaling method is used, it is mainly through adding acidic drugs, which has the advantage of high cleaning efficiency and an obvious effect. However, acidic agent corrosion 
not only shortens the life of heat transfer equipment but also causes second pollution and other unavoidable shortcomings [5-8]. Traditional physical descaling methods such as scraping, sand blasting, and metal or nylon brushing incur downtime and repair costs [9]. For these reasons, new physical methods such as electronic anti-fouling technology, catalytic material, and sudden pressure change have recently been developed [5-7].

Electronic anti-fouling technology (EAFT) is a new kind of physical descaling method that can be used in descaling of recycled cooling water system without polluting and high consumption [10]. Generally, the system consists of an EAFT control unit, a solenoid coil, and a pipe. The control unit provides alternating current in the coil, which is tightly wrapped on the outside of the pipe, and the solenoid coil can motivate induction magnetic field in the pipe. As depicted by Faraday's law, the alternating induction magnetic field can generate an induced electric field in the pipe. Under the action of the induced electric field, the sparingly soluble salts form precipitates before entering the heat transfer equipment, so that the degree of supersaturation of sparingly soluble salts is reduced before entering the heat exchanger [6, 11-12]. In addition, the generated scale under the EAFT treatment is soft and with poor adhesion ability, which is easy to be washed away by the water flow within the pipe $[8,13-14]$.

Liu et al. [15] found that the fouling resistance in the pipe with the EAFT treatment had significant decline at the end of 4 days' test, which was $46 \%$ less than the one without the EAFT treatment. Piyadasa et al. [11] investigated the precipitation characteristics of calcium carbonate under the influence of the EAFT treatment, and the results showed that the EAFT treatment can influence both the profile of calcium carbonate precipitation and the morphology of the resulting microcrystals, enhanced particle coagulation in the solution. Kim et al. [16] combined use of EAFT and filtration technology, finding that when filtration was used in conjunction with the EAFT, a substantially better fouling mitigation was obtained, as the filtration removed the mineral particles that were produced by the EAFT. Zhang et al. [17] investigated the effect of the EAFT in a closed cooling tower and found that fouling resistance in the case with and without EAFT treatment increased by $7.51 \%$ and $25.14 \%$, respectively, at the end of $214 \mathrm{~h}$ of tests. All the above tests demonstrate that the EAFT treatment can effectively reduce the fouling in actual heat exchangers.

Lee et al. [18] studied the effect of flow velocity on the EAFT and found that as flow velocity increased, the benefit of the in-line EAFT treatment decreased. But there is no related experimental study that shows how the output frequency of EAFT control unit, winding number of coil, and pipe diameter impact the descaling, which is considered to be as important as the flow velocity in electronic anti-fouling technology.

Jiang et al. [19-20] experimented and discovered that with the increase of magnetic induction intensity, the descaling effect of EAFT treatment improves.
The magnetic induction intensity is determined by the current in the solenoid coil, which can be explained by Ampere's loop theorem and Faraday's rule. This means that the descaling effect is indirectly decided by the size of current in the solenoid coil. In other words, the current in coil can be used to characterize the anti-fouling effect of the EAFT.

In summary, the current and the magnetic induction intensity are the significant parameters that affect the effect of EAFT treatment directly, and the output frequency of EAFT control unit, winding number of coils, and pipe diameter are important output parameters of EAFT treatment. So further investigation on the change rule of current and magnetic induction intensity with respect to the number of turns, diameter, and frequency at a given voltage need to be developed.

Accordingly, the objective of the present study is to explore how frequency, the number of turns, and winding diameter affect the current in the solenoid coil and magnetic induction intensity in PVC pipes. Results of this study described the change rule of current and magnetic induction intensity in PVC pipes affected by various factors, which not only put forward an excellent processing parameter of the electronic anti-fouling technology, but also provide design guidance for the EAFT control unit.

\section{Material and Methods}

\section{Material}

Fig. 1 shows a schematic diagram of test facilities that consist of the EAFT control unit, pipe, solenoid coil, series resistance, and oscilloscope. The wire is tightly wrapped around the pipe to form a solenoid coil, with one end of the solenoid coil directly connected to the control unit, and the other indirectly connected to the EAFT control unit through series resistance. The hardness of water in the pipe was artificially configured to $1,000 \mathrm{ppm}$ and the solution was in a static state.

If a non-magnetic pipe is used, there is a different distribution of the induction magnetic compared with magnetic pipe [21]. The EAFT with non-magnetic pipe

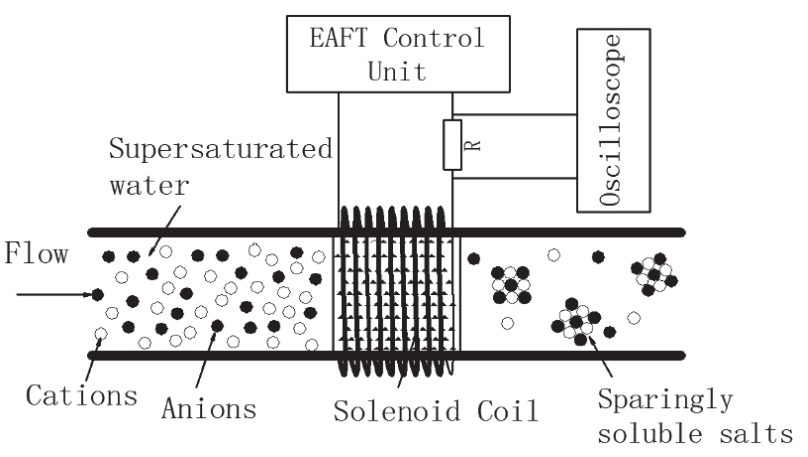

Fig. 1. Schematic drawing of the experimental device. 


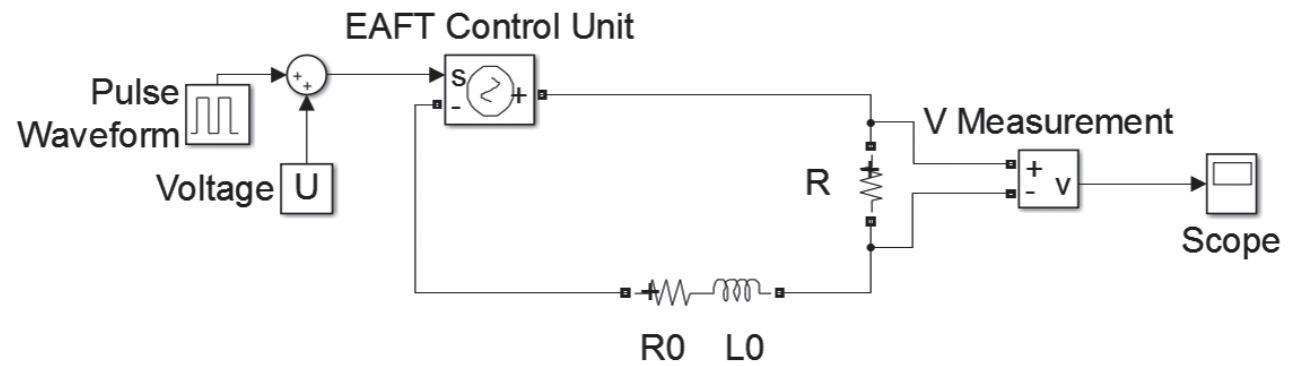

Fig. 2. Diagram of experimental device equivalent circuit.

is studied in this paper and the material of the water pipe is PVC.

The EAFT control unit produces a square wave that can maximize induction [6]. PVC pipes with different diameters are used in the experiment. Assume that the diameter of the solenoid coil is the diameter of the pipe. The type of wire is polyvinyl chloride-insulated flexible cables with copper core meeting the Chinese National Standard. The wire is $5.3 \mathrm{~mm}$ in diameter and $0.8 \mathrm{~mm}$ in insulation thickness. Series resistance in the experiment has the stable high frequency characteristic. The model of the oscilloscope is TBS1102 produced by Tektronix and it is $100 \mathrm{MHz}$ in bandwidth, $\pm 3 \%$ in vertical precision.

\section{Methods}

Fig. 2 shows the equivalent circuit diagram of the experiment. The operating frequency of the EAFT control unit is almost less than $1 \mathrm{MHz}$, while the solenoid coil can be equivalent to ideal inductance L0 and resistance R0 in series [22], so the solenoid coil in Fig. 2 is converted into the resistance $\mathrm{R} 0$ and the ideal inductance L0 being in series. Resistance $\mathrm{R}$ in series is used to obtain the current in the circuit. V measurement and scope are combined to represent the oscilloscope for measuring voltage across the series resistor.

This experiment sets the output voltage of the EAFT control unit on $9.6 \mathrm{~V}$, and the frequency ranges from $2 \mathrm{kHz}-27 \mathrm{kHz}$. The turns of the solenoid coil wrapped around each PVC pipe are 30, 50, 70, and 90, respectively. The diameters of the 4 PVC pipes are $100 \mathrm{~mm}, 160 \mathrm{~mm}$, $200 \mathrm{~mm}$, and $315 \mathrm{~mm}$. The voltage of the series resistor under different parameters can be measured by the oscilloscope.

According to the voltage measured at the 2 ends of the series resistance and the value of resistance, current in the circuit can be calculated by Ohm's law:

$$
I=\frac{U}{R}
$$

...where $U$ is the voltage between the 2 ends of series resistance and $\mathrm{R}$ is the value of resistance.

Based on the obtained current value, the magnetic induction intensity in the midpoint of the solenoid coil can be calculated by:

$$
B=\frac{\mu_{0} n I}{2} \frac{l_{0}}{\left(l_{0}^{2} / 4+r_{0}^{2}\right)^{1 / 2}}
$$

...where $\mu_{0}$ is magnetic permeability of solution, $\mathrm{n}$ is the turns of solenoid coil, I is current in the circuit, $1_{0}$ is the length of solenoid coil, and $r_{0}$ is the radius of solenoid coil.

The current and the magnetic induction intensity in different parameters can be obtained by changing

a)

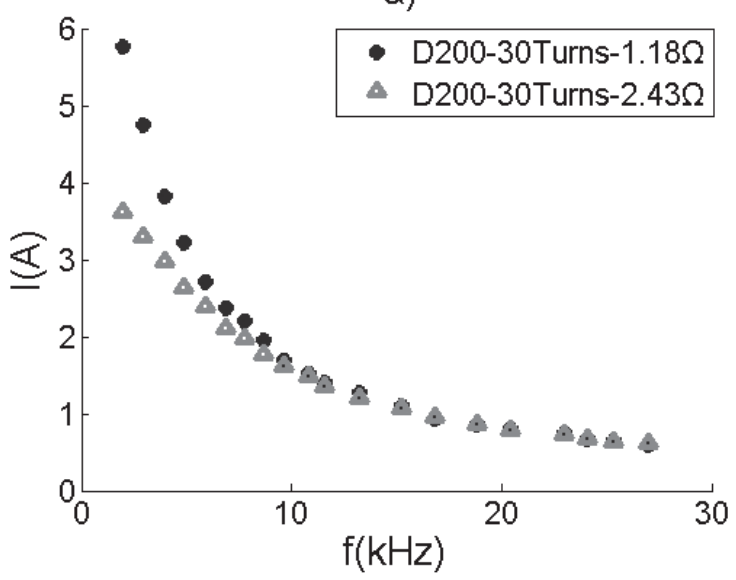

b)

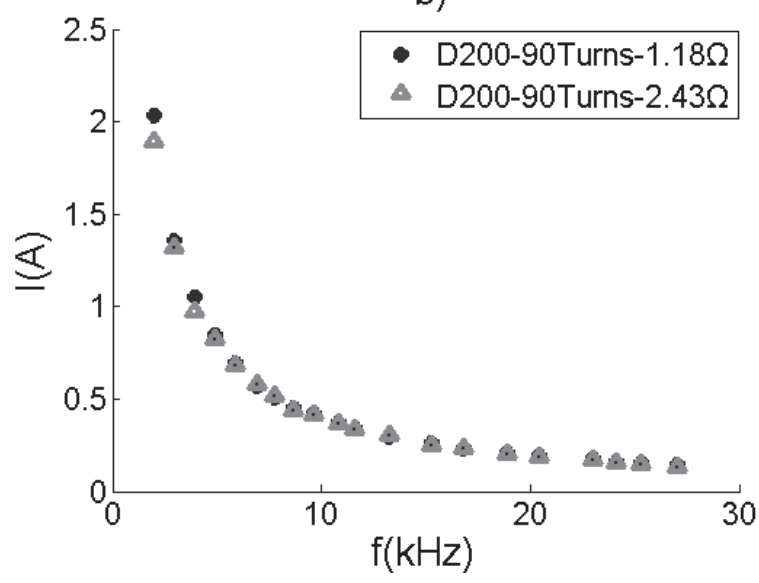

Fig. 3. The effect of series resistance on measuring current: a) diameter $100 \mathrm{~mm}$ under the turns of 30 and b) diameter $200 \mathrm{~mm}$ under the turns of 90 . 
the frequency, winding turns, winding diameter. Thus, we can get the change rule of current and magnetic induction with respect to different frequency, the number of turns, and pipe diameter.

The original series circuit with a resistor will certainly impact the current of the circuit. In order to analyze the effect of series resistance on the circuit, the current in coil winding with 30 turns and 90 turns are measured by using the resistance of $1.18 \Omega$ and the resistance of $2.43 \Omega$, respectively. As shown in Fig. 3, when the resistance is connected in series, the change rules of current remain the same. Because the error of the two curves is quite small, the influence of series resistance can be neglected when analyzing the change rules.

The greater value of series resistance, the more the effect of alternating current value in the circuit. So in the later experiment, resistance of $1.18 \Omega$ was used to reduce the error. Meanwhile, the value of resistance is assumed to be a constant value because it is little varied with frequency. This paper has only discussed the influence of the factors on the change rule of the current and the magnetic induction intensity. Research on reducing the influence of series resistance on current will be described in future research.

\section{Results and Discussion}

Figs 4-6 respectively show the change rule of current and magnetic induction intensity with respect to different frequency, turns, and diameter.

\section{Change Rule of Current and Magnetic Induction Intensity with Respect to Frequency}

Fig. 4a) shows the change rule of alternating current with respect to frequency. The alternating current in the coil decreases with the increase of frequency, and there is a smaller decline with the increase of frequency. For example, in the case of 70 turns coil winding around the diameter of $100 \mathrm{~mm}$ PVC pipe, the alternating current decreases from 5.5 A to $0.62 \mathrm{~A}$ as the frequency increases from $2 \mathrm{kHz}$ to $27 \mathrm{kHz}$. The current decreases from 5.5 A to $1.13 \mathrm{~A}$ as the frequency increases from $2 \mathrm{kHz}$ to $14.5 \mathrm{kHz}$, and the current decreases from $1.13 \mathrm{~A}$ to $0.62 \mathrm{~A}$ when there is the same drop amplitude of frequency. As shown in Fig. 4a), both different turns and diameter show the same change rule of current with respect to frequency. The reason is the increase in total impedance of the coil as the frequency increases, resulting in a decrease in the current under constant voltage output.

Fig. 4b) shows the change rule of magnetic induction intensity with respect to frequency. It can be seen from Fig. 4b) that magnetic induction intensity decreases with the increase of the frequency, and the drop amplitude decreases with increase of the frequency, which is similar to the change rule of the current with frequency. For example, in the case of a 70-turns coil winding around the diameter of $200 \mathrm{~mm}$ PVC pipe, the magnetic induction intensity decreases from $0.22 \mathrm{mT}$ to $0.014 \mathrm{mT}$ as the frequency increases from $2 \mathrm{kHz}$ to $27 \mathrm{kHz}$. The magnetic induction intensity decreases from $0.22 \mathrm{mT}$ to $0.027 \mathrm{mT}$ when the frequency increases from $2 \mathrm{kHz}$ to $14.5 \mathrm{kHz}$, and the magnetic induction intensity decreases from $0.027 \mathrm{mT}$ to $0.014 \mathrm{mT}$ when there is the same drop in amplitude of frequency. We can conclude from Fig. 4b) that both different turns and diameter show the same change rule of magnetic induction intensity with respect to frequency. When the output frequency of the EAFT control unit changes, only the current in the many factors that affect magnetic induction intensity changes. And when other factors remain unchanged, the magnetic induction intensity is proportional to the current. Therefore, the change rule of magnetic induction with frequency is similar to the change rule of current with frequency.
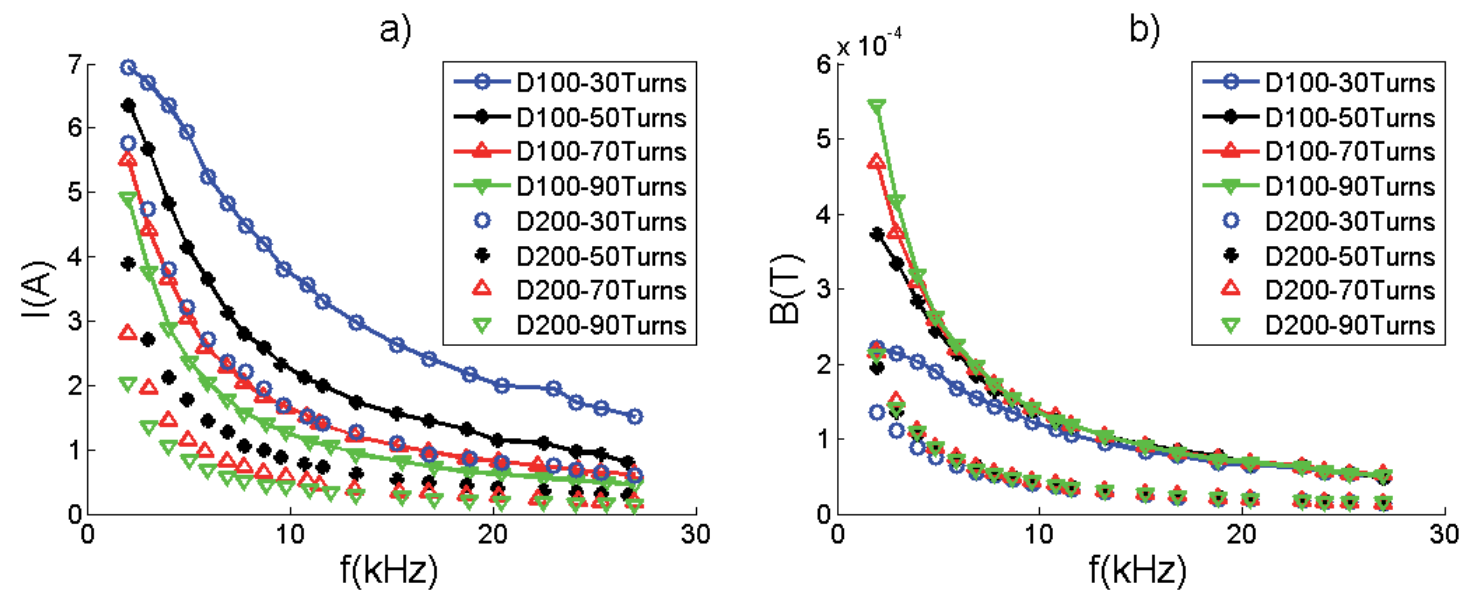

Fig. 4. Change of current and magnetic induction with frequency: a) current and b) magnetic induction - both under diameter of $100 \mathrm{~mm}$ and $200 \mathrm{~mm}$, and turns of 30, 50, 70, and 90 . 
Change Rule of Current and Magnetic Induction Intensity with Respect to Number of Turns

Fig. 5a) shows the change rule of current with respect to turns. The current decreases as the number of turns increases, both under different frequency and diameter show the same change rule. For example, the current is $1.102 \mathrm{~A}, 0.542 \mathrm{~A}, 0.342 \mathrm{~A}$, and $0.263 \mathrm{~A}$ under turns of 30 , 50,70 , and 90 , respectively, when the output frequency of EAFT control unit is $15 \mathrm{kHz}$ and pipe diameter is 200 $\mathrm{mm}$. In this case, winding coil increased from 30 turns to 90 turns, the current value decreased by $76.1 \%$. It can be seen from the figure that the change rule of current is not a simple linear relationship with respect to turns. The main reason for the change rule of current is that total impedance increases with the number of turns, which leads to a decrease in the current.

The magnetic induction intensity in different frequencies of $5 \mathrm{kHz}, 10 \mathrm{kHz}, 15 \mathrm{kHz}$, and $20 \mathrm{kHz}$ is shown in Fig. 5b), which shows that when the frequency is greater than $10 \mathrm{kHz}$, the magnetic induction intensity basically does not change with the increase of turns. For example, the magnetic induction is $0.026 \mathrm{mT}, 0.027 \mathrm{mT}$, $0.027 \mathrm{mT}$, and $0.027 \mathrm{mT}$ under turns of $30,50,70$, and 90 , respectively, when the output frequency of EAFT control unit is $15 \mathrm{kHz}$ and the pipe diameter is $200 \mathrm{~mm}$. With the increase in the number of turns, the magnetic induction basically remains unchanged. The slight change of the magnetic induction intensity with turns in the lower frequency is due to the error caused by the introduction of series resistance. When the frequency is relatively high, the total impedance of the coil becomes larger and the influence of the series resistance on the circuit is smaller at the same time. It can be seen from Eq. (2) that the size of the magnetic induction intensity is determined by current value in the coil, length of solenoid coil, radius of solenoid coil, turns of solenoid coil, and so on. When the turns of the winding coil change, it will inevitably change the current value in the winding coil and the length of the winding coil. Changes in various factors eventually cause the magnetic induction intensity to remain unchanged, but the increase of winding coil will increase the processing time of the electromagnetic descaling system and make the descaling effect better.

\section{Change Rule of Current and Magnetic Induction Intensity with Respect to Diameter}

Fig. 6a) presents the change of current with 4 different diameters in 4 different frequencies. Fig. 6a) shows that the current in coil wrapped around the diameter of 100 $\mathrm{mm}$ is larger than other 3 diameters, and the current decreases as the diameter increases. For example, in the case of winding turns of 30 and output frequency of $10 \mathrm{kHz}$, the current is $3.814 \mathrm{~A}, 2.203 \mathrm{~A}, 1.695 \mathrm{~A}$, $0.966 \mathrm{~A}$ under pipe diameter of $100 \mathrm{~mm}, 160 \mathrm{~mm}$, $200 \mathrm{~mm}$, and $315 \mathrm{~mm}$, respectively, as the diameter of PVC pipe increased from $100 \mathrm{~mm}$ to $315 \mathrm{~mm}$, the current value dropped by $74.7 \%$. Because the increase in the diameter leads to an increase in total impedance, the increase in total impedance results in a decrease in the current. This phenomenon is the same in the 30 turns and the 90 turns of the winding as shown in Fig. 6a).

Fig. 6b) shows the change of magnetic induction intensity with 4 different diameters in different frequencies. It can be seen from Fig. 6b) that the magnetic induction intensity in the coil wrapped around the diameter of $100 \mathrm{~mm}$ is larger than other 3 diameters, magnetic induction decreases as diameter increases. Both under different turns and frequency show that magnetic induction decreases as diameter increases. For example, in the case of winding turns of 30 and output frequency of $10 \mathrm{kHz}$, magnetic induction is $0.122 \mathrm{mT}$, $0.059 \mathrm{mT}, 0.04 \mathrm{mT}$, and $0.016 \mathrm{mT}$ under pipe diameter of $100 \mathrm{~mm}, 160 \mathrm{~mm}, 200 \mathrm{~mm}$, and $315 \mathrm{~mm}$, respectively, and as the diameter of PVC pipe increased from $100 \mathrm{~mm}$ to $315 \mathrm{~mm}$, the magnetic induction intensity dropped by $86.9 \%$. Although the magnitude of magnetic induction is determined by a number of factors, the increase in diameter leads to a decrease in current in the loop and an increase in coil-winding diameter, both of which result in a decrease in magnetic induction. a)

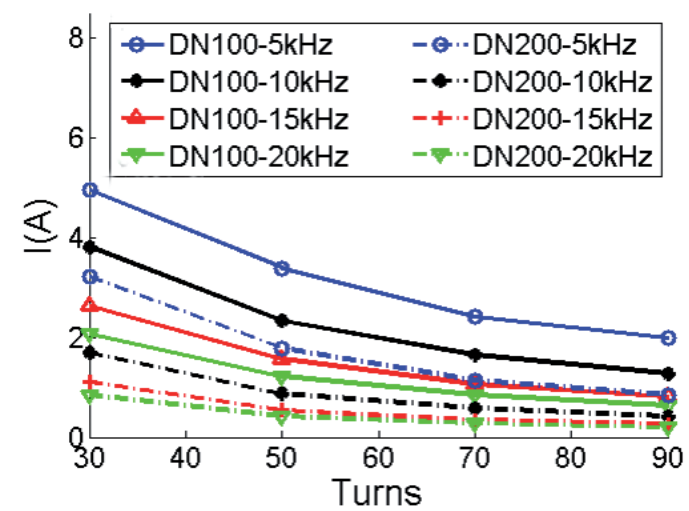

b)

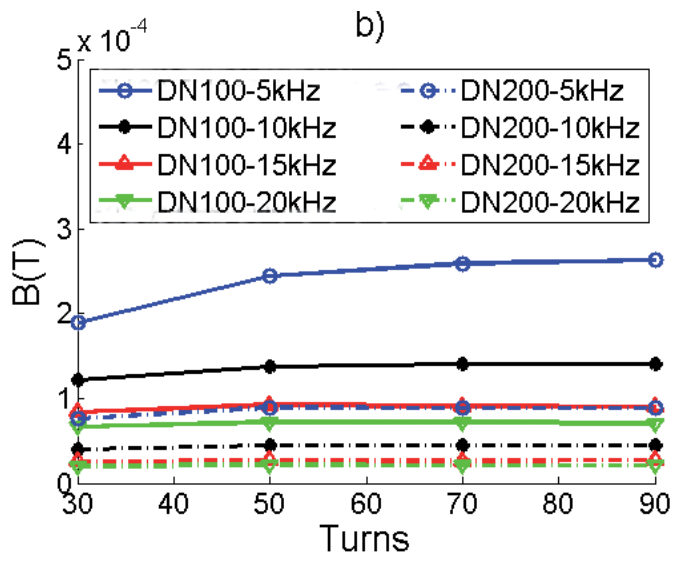

Fig. 5. Change of current and magnetic induction with the number of turns: a) current and b) magnetic induction both under diameter of $100 \mathrm{~mm}$ and $200 \mathrm{~mm}$, frequency of $5 \mathrm{kHz}, 10 \mathrm{kHz}, 15 \mathrm{kHz}$, and $20 \mathrm{kHz}$. 

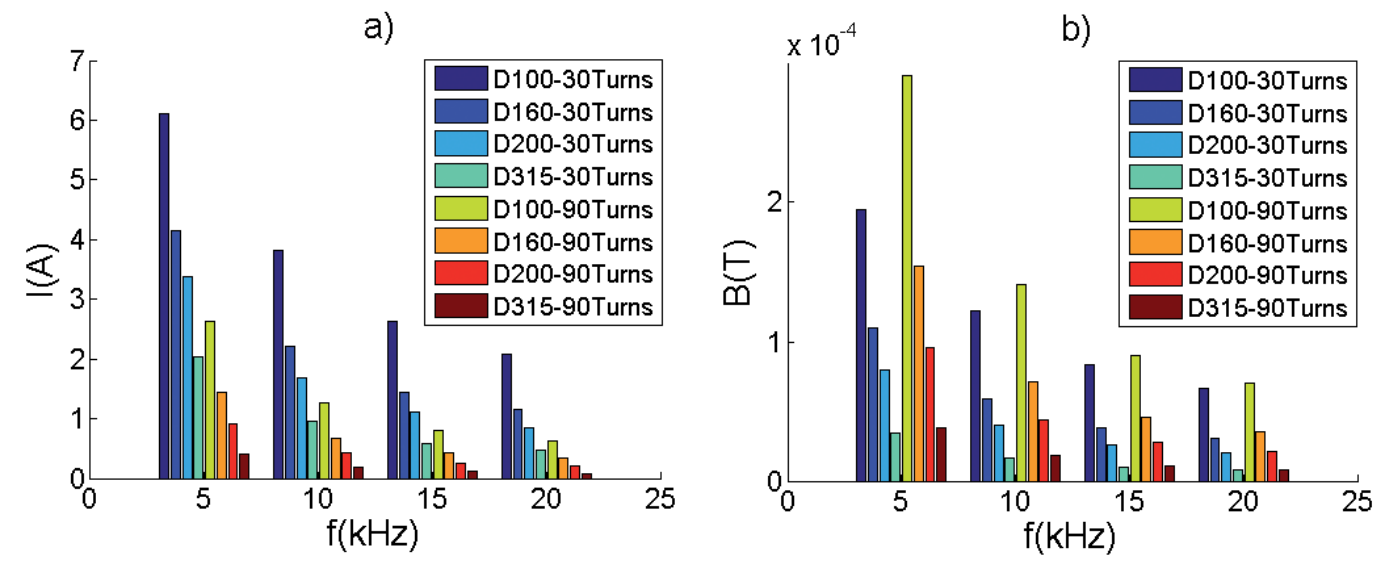

Fig. 6. Change of current and magnetic induction with diameter: a) current and b) magnetic induction both under turns of 30 and 90 , and diameter of $100 \mathrm{~mm}, 160 \mathrm{~mm}, 200 \mathrm{~mm}$, and $315 \mathrm{~mm}$.

Through the analysis of experimental data, it can be concluded that the current in the coil decreases with the increase of frequency, number of turns, and pipe diameter, respectively. Magnetic induction intensity basically does not change with the increase of turns, and it decreases as the frequency and the diameter increase. In the process of exploitation, changes in pipe diameter often occur. When the diameter of the pipe increases, the magnetic induction intensity in the pipe will be reduced and the effect of the descaling is reduced. Then the output frequency of the control system should be decreased to increase the magnetic induction in the pipe and the number of turns should be increased to increase the processing time of the descaling system, so as to improve the descaling effect.

Comparing the magnetic induction intensity under various frequencies, number of turns, and diameters in the experiments, we find that when PVC pipe is used, the output frequency of the EAFT control unit is $2 \mathrm{kHz}$, the diameter of pipe is $100 \mathrm{~mm}$, and the number of turns is 90 , causing the effect of EAFT treatment to be better.

\section{Conclusions}

Non-magnetic pipe, PVC, is used in this experiment. The change rule of the current in the solenoid coil and the magnetic induction intensity in the midpoint of the solenoid coil, with respect to every parameter, were observed in the experiment. The current is obtained by measuring the voltage of the series resistance, and then the magnetic induction can be calculated by formula. The key conclusions of the experiments are organized as follows:

1) Alternating current and magnetic induction intensity decrease with the increase of frequency, and the drop amplitude in both cases decrease as frequency increases.

2) Current in the coil decreases as the number of turns increases. The magnetic induction intensity remains unchanged as the number of turns increases, which is different from the change rule of the current.

3) Current and magnetic induction intensity decrease as diameter increases.

4) When PVC pipe is used, the output frequency of EAFT control unit is $2 \mathrm{kHz}$, the diameter of pipe is $100 \mathrm{~mm}$, and the number of turns is 90 , the effect of EAFT treatment improves.

\section{Acknowledgements}

This work is supported by the National Key R\&D Program of China (2017YFB0603300). Thanks are due to $\mathrm{Hu}$ Duan for assistance with the experiments and to Kui Cao and Baohua Liang for valuable discussions. We are also grateful to Mengmeng Zhang and Shaoyi Wang for insightful and constructive comments that greatly improved this manuscript.

\section{Conflict of Interest}

The authors declare no conflict of interest.

\section{References}

1. PODGÓRNI E., BOGUNIEWICZ-ZABŁOCKA J., KŁOSOK-BAZAN I. The impact of nano-silver dose on microorganism-deactivation effectiveness in water circulating in cooling tower cycle. Polish Journal of Environmental Studies, 24 (5), 2321, 2015.

2. NUR S.Z., JOHAN S., KHALIDA M., MIKA S. Magnetic field application and its potential in water and wastewater treatment systems. Separation \& Purification Reviews, $\mathbf{4 3}$ (3), 206, 2014.

3. QUAN Z.H., CHEN Y.C., MA C.F., WANG C.M., LI B. Experimental Study on Anti-Fouling Performance in a Heat Exchanger with Low Voltage Electrolysis Treatment. Heat Transfer Engineering, 30 (3), 181, 2009. 
4. KAZI S.N., TENG K.H., ZAKARIA M.S., SADEGHINEZHAD E., BAKAR M.A. Study of mineral fouling mitigation on heat exchanger surface. Desalination, 367, 248, 2015.

5. LE D.T., REN F., ZHANG M. Physical water treatment using RF electric fields for the mitigation of $\mathrm{CaCO}_{3}$ fouling in cooling water. International Journal of Heat \& Mass Transfer, 53 (7), 1426, 2010.

6. CHO Y.I., FAN C., CHOI B.G. Use of electronic antifouling technology with filtration to prevent fouling in a heat exchanger. International Journal of Heat \& Mass Transfer, 41 (41), 2961, 1998.

7. XUEFEI M., LAN X., JIAPENG C., ZIKANG Y., WEI $\mathrm{H}$. Experimental study on calcium carbonate precipitation using electromagnetic field treatment. Water Science \& Technology A Journal of the International Association on Water Pollution Research, 67 (12), 2784, 2013.

8. ZHAO J.D., LIU Z.A., ZHAO E.J. Combined effect of constant high voltage electrostatic field and variable frequency pulsed electromagnetic field on the morphology of calcium carbonate scale in circulating cooling water systems. Water Science \& Technology A Journal of the International Association on Water Pollution Research, 70 (6), 1074, 2014.

9. TIJING L.D., PAK B.C., LEE D.H., CHO Y.I. Heat-treated titanium balls for the mitigation of mineral fouling in heat exchangers. Experimental Heat Transfer, 21 (2), 115, 2008.

10. DOELMAN J.D.B. Electronic water treatment is an environmentally friendly alternative to chemical and mechanical descaling. Pollution Engineering, 46 (4), 33, 2014.

11. PIYADASA C., RIDGWAY H.F., YEAGER T.R., STEWART M.B., PELEKANI C., GRAY S.R., ORBELL J.D. The application of electromagnetic fields to the control of the scaling and biofouling of reverse osmosis membranes - a review. Desalination, 418, 19, 2017.

12. PIYADASA C., YEAGER T.R., GRAY S.R., STEWART M.B., RIDGWAY H.F., PELEKANI C., ORBELL J.D. The influence of electromagnetic fields from two commercially available water-treatment devices on calcium carbonate precipitation. Environmental Science Water Research \& Technology, 3, 566, 2017.
13. NASSER W.N.A., RUWAIE A.H.A., HOUNSLOW M.J., SALMAN A.D. Influence of electronic antifouling on agglomeration of calcium carbonate. Powder Technology, 206 (1), 201, 2011.

14. TRUEBA A., GARCÍA S., OTERO F.M., VEGA L.M., MADARIAGA E. The effect of electromagnetic fields on biofouling in a heat exchange system using seawater. Biofouling, 31 (1), 19, 2015.

15. LIU R., CHO Y.I. Combined use of an electronic antifouling technology and brush punching for scale removal in a water-cooled plain tube. Experimental Heat Transfer, 12 (3), 203, 1999.

16. KIM W., CHO Y.I. Benefit of filtration in physical water treatment for the mitigation of mineral fouling in heat exchangers. International Communications in Heat \& Mass Transfer, 38 (8), 1008, 2011.

17. ZHANG L.X., CHEN Y.B., GAO M., LI X., LIN Z.H. Validation of electronic anti-fouling technology in the spray water side of evaporative cooler. International Journal of Heat \& Mass Transfer, 93, 624, 2016.

18. LEE S.H., CHO Y.I. Velocity effect on electronicantifouling technology to mitigate mineral fouling in enhanced-tube heat exchanger. International Journal of Heat \& Mass Transfer, 45 (20), 4163, 1998.

19. JIANG D.N., ZEWDU S., FU R.L. Experimental parameter study on prevention from descaling. Journal of Tianjin University of Science \& Technology, 23 (3), 64, 2008.

20. JIANG D.N., ZHOU K. Experimental study on antiscale of pipes based on alternating magnetic field. Journal of TianJin University of Technology and Education, 18 (1), 21, 2008.

21. HAN Y., ZHAO Y., CHAI X. Mathematical modeling of induced current for electronic anti-fouling system and optimizing of the system parameters. International Journal of Applied Electromagnetics \& Mechanics, 40 (3), 237, 2012.

22. CHEN G.J., CHEN K., ZHOU Y.P., LI B. Inductor frequency characteristic and its application in RLC series resonant circuit. College Physics, 35 (6), 29, 2016. 
